\title{
Ultrarelativistic electron-positron plasma
}

\author{
M.H. Thoma ${ }^{a}$
}

Max-Planck-Institut für extraterrestrische Physik, Gießenbachstr., 85748 Garching, Germany

Received 6 October 2008 / Received in final form 19 January 2009

Published online 13 March 2009 - (C) EDP Sciences, Società Italiana di Fisica, Springer-Verlag 2009

\begin{abstract}
Ultrarelativistic electron-positron plasmas can be produced in high-intensity laser fields and play a role in various astrophysical situations. Their properties can be calculated using QED at finite temperature. Here we will use perturbative QED at finite temperature for calculating various important properties, such as the equation of state, dispersion relations of collective plasma modes of photons and electrons, Debye screening, damping rates, mean free paths, collision times, transport coefficients, and particle production rates, of ultrarelativistic electron-positron plasmas. In particular, we will focus on electron-positron plasmas produced with ultra-strong lasers.
\end{abstract}

PACS. 52.27.Ep Electron-positron plasmas - 11.10.Wx Electron-positron plasmas

\section{Introduction}

Plasmas, i.e. (partly) ionized gases, are considered to be the fourth state of matter after the solid, liquid, and gaseous states. The plasma state dominates in the observable Universe: $99 \%$ of the visible matter is in the plasma state, namely in the form of stars and hot interstellar and intergalactic gases. Plasmas emit light due to the excitation of atoms and ions and recombination. Plasmas can be produced by high temperatures, such as in the sun or fusion reactors, by electric fields (discharges), as used for illumination in neon tubes or in lightening, or by radiation, such as in the Crab nebula where the pulsar in the center emits synchrotron radiation.

Plasmas can be classified according to various aspects: relativistic plasmas, e.g. the electron-positron plasma in a supernova explosion, are plasmas in which the thermal energy $k_{B} T$ of the plasma particles is of the order of their rest mass energy $m c^{2}$ or larger. Quantum plasmas, e.g. the degenerate electron component in a white dwarf, are plasmas in which the thermal de Broglie wave length $\lambda_{B}=h /\left(m v_{t h}\right)$ is of the order of the interparticle distance $d$ or larger. Here $v_{t h}$ is the thermal velocity of the particles. Strongly coupled plasmas, e.g. the ion component in white dwarfs, are plasmas in which the interaction energy between the particles is larger than their thermal energy. In non-relativistic plasmas this corresponds to a Coulomb coupling parameter $\Gamma_{C}=Q^{2} /(d k T)>1$.

Plasmas in nature, like in comets, in aurorae, in the corona of the sun, in lightening, in flames, or in the sun, and in technology, like in discharges or fusion reactors, cover a wide range of pressures and temperatures. All of

\footnotetext{
a e-mail: thoma@mpe.mpg.de
}

these plasmas are non-relativistic, classical, and weakly coupled systems.

Electron-positron plasmas (EPPs) are created in the presence of strong electric or magnetic fields or extremely high temperatures, where massive pair production sets in. For example in a supernova explosion temperatures up to $3 \times 10^{11} \mathrm{~K}$ corresponding to $k_{B} T \simeq 30 \mathrm{MeV} \gg 2 m_{e} c^{2}=$ $1.022 \mathrm{MeV}$ will lead to an ultrarelativistic EPP [1]. Also in the vicinity of magnetars, i.e. neutron stars with magnetic fields $B>10^{14} \mathrm{G}$, and in accretion disks around black holes EPPs show up.

Recently the possibility to create ultrarelativistic EPPs with high-intensity lasers $\left(I>10^{18} \mathrm{~W} / \mathrm{cm}^{2}\right)$ have been discussed. For example two opposite laser pulses hitting a thin gold foil will heat up the electrons in the foil up to several MeV leading to pair creation [2].

In the following we will discuss the properties of an ultrarelativistic EPP using quantum field theory (QED) at finite temperature. We will follow closely the review article [3]. We will not discuss the production mechanism and equilibration of the EPP here.

\section{Field theoretic description of an electron-positron plasma}

Throughout the paper we will use natural units, i.e. $\hbar=$ $c=k_{B}=1$, as usual in quantum field theory, in which all units are given in powers of $\mathrm{MeV}$. The conversion to conventional units can be achieved by $1=\hbar c=1.97 \times$ $10^{-13} \mathrm{MeV} m$ from which $1 \mathrm{MeV}=1.60 \times 10^{-13} \mathrm{~J} \hat{=} 5.08 \times$ $10^{12} \mathrm{~m}^{-1} \hat{=} 1.52 \times 10^{21} \mathrm{~s}^{-1}$ follows. In these units the electron charge $e=0.3$ corresponding to a fine structure constant $\alpha=e^{2} /(4 \pi)=1 / 137$. 


\subsection{Equation of state}

We will start with the equation of state of an EPP and compute it under the following assumptions:

1. ultrarelativistic EPP, i.e. $T \gg m$,

2. thermal and chemical equilibrium,

3. equal electron and positron density, i.e. vanishing chemical potential,

4. ideal gas, i.e. no interactions in the plasma,

5. infinitely extended, homogeneous, and isotropic EPP.

We will relax some of these assumptions in the following sections. According to these assumptions the distribution function of the electrons and positrons is given by the Fermi-Dirac distribution

$$
n_{F}(p)=\frac{1}{e^{p / T}+1}
$$

and of the photons by the Bose-Einstein distribution

$$
n_{F}(p)=\frac{1}{e^{p / T}-1}
$$

where the momentum $p$ is identical to the energy $E$ of the particles in the ultrarelativistic case. It should be noted that the photons are in equilibrium with electrons and positrons under the above assumptions, i.e. the system is actually an electron-positron-photon gas.

The particle and energy density can be calculated by integrating over the distribution functions. The particle number density of the electrons and positrons follows from integrating over the Fermi-Dirac distribution as

$$
\rho_{e}^{e q}=g_{F} \int \frac{d^{3} p}{(2 \pi)^{3}} n_{F}(p)=\frac{3}{\pi^{2}} \zeta(3) T^{3}=0.37 T^{3},
$$

where $g_{F}=4$ is the number of degrees of freedom corresponding to the electrons and positrons in the two spin states. Assuming a temperature of $T=10 \mathrm{MeV}$, we find $\rho_{e}^{e q}=370 \mathrm{MeV}^{3}=4.9 \times 10^{40} \mathrm{~m}^{-3}$.

The photon density follows accordingly by integrating over the Bose-Einstein distribution with $g_{B}=2$ degrees of freedom corresponding to the two polarization states as $\rho_{p h}^{e q}=\left(2 / \pi^{2}\right) \zeta(3) T^{3}=0.24 T^{3}$.

The energy density of the electron-positron-photon gas is obtained from

$$
\begin{aligned}
\epsilon^{e q} & =g_{F} \int \frac{d^{3} p}{(2 \pi)^{3}} p n_{F}(p)+g_{B} \int \frac{d^{3} p}{(2 \pi)^{3}} p n_{B}(p) \\
& =\frac{11 \pi^{2}}{60} T^{4}=1.81 T^{4}
\end{aligned}
$$

where the photons contribute $36 \%$ to the energy density. Here the Boltzmann law, $\epsilon^{e q} \sim T^{4}$, holds also for the fermions because we neglected their masses.

For $T=10 \mathrm{MeV}$ we find $\epsilon^{e q}=3.8 \times 10^{29} \mathrm{~J} \mathrm{~m}^{-3}$ In a volume of $10^{12} \mathrm{~m}^{3}$ (corresponding to the size of a neutron star) the total thermal energy of the EPP is $3.8 \times 10^{41} \mathrm{~J}$, which corresponds to about $10 \%$ of the entire energy (without neutrinos) released in a supernova type
II explosion. In a volume of $1 \mu \mathrm{m}^{3}$ there is still an energy of $3.8 \times 10^{11} \mathrm{~J}$ contained. It is hard to imagine that such an energy can be provided by a laser experiment where a more dilute, i.e. non-equilibrium, EPP is expected (see Sect. 2.4).

The Coulomb coupling parameter of the EPP, which is a measure for the non-ideal behavior of a plasma [4], is given by $\Gamma_{C}=e^{2} /(d T)$, where $d \simeq \rho_{e}^{e q-1 / 3}=2.7 \times$ $10^{-14} \mathrm{~m}$ is the interparticle distance. For $T=10 \mathrm{MeV}$ we find $\Gamma_{C}=5.3 \times 10^{-3}$ which shows that the EPP is a weakly coupled plasma. Therefore the ideal gas results for the equation of state derived above are a good approximation. After all, interactions in the EPP play an important role, for example, for the collective behavior of the plasma as discussed in the next section and for equilibration of the plasma. Obviously, the interactions can be treated by perturbation theory.

\subsection{Collective phenomena}

Collective effects in a plasma are associated with longrange interactions within the plasma. Important examples are Debye screening and plasma waves. In non-relativistic ion-electron plasmas [5] plasmas these phenomena can be described by classical transport theory (Vlasov equation). For example, the electron plasma frequency reads

$$
\omega_{p l}=\sqrt{\frac{4 \pi e^{2} \rho_{e}}{m_{e}}}
$$

and the Debye screening length due to the electrons in the plasma

$$
\lambda_{D}=\sqrt{\frac{k_{B} T_{e}}{4 \pi e^{2} \rho_{e}}}
$$

where $\rho_{e}$ is the electron number density, $T_{e}$ the temperature of the electron component, and $m_{e}$ the electron mass. In an ultrarelativistic plasma with $T \gg m$ the masses can be neglected and the important scales are the temperature $T$, called the hard scale, and the soft scale $e T$, which determines the collective phenomena as we will see below.

Interactions between relativistic electrons and positrons can be treated by using perturbative QED. This corresponds to an expansion in the fine structure constant $\alpha$. Most conveniently Feynman diagrams are considered from which via Feynman rules quantities such as scattering cross sections, decay and production rates, or life times can be calculated directly. In an EPP the interactions take place in the presence of a heat bath. Hence we have to consider QED at finite temperature. For this purpose the Feynman rules are extended to finite temperature which can be achieved by using the imaginary or real time formalism [6,7]. The calculations are similar to the ones done already within the last 30 years for the properties of the quark-gluon plasma using perturbative QCD at finite temperatures. As a matter of fact, many results from the quark-gluon plasma (see e.g. Ref. [8]) can be directly carried over to the EPP. 


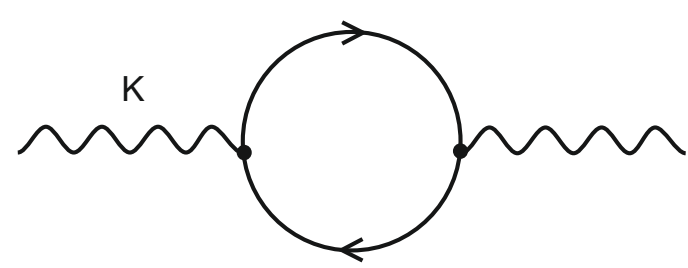

Fig. 1. One-loop polarization tensor.

An important quantity is the polarization tensor or photon self-energy. The lowest order diagram for the polarization tensor is shown in Figure 1. Assuming the external four momentum $K=\left(k_{0}, \mathbf{k}\right)$ to be soft, i.e. the frequency $k_{0}=\omega$ and $k=|\mathbf{k}|$ to be much smaller than $T$, and the internal loop momenta to be hard, an analytic result can be found using the real or imaginary time formalism $[9,10]$

$$
\begin{aligned}
& \Pi_{L}(\omega, k)=-3 m_{p h}^{2}\left(1-\frac{\omega}{2 k} \ln \frac{\omega+k}{\omega-k}\right) \\
& \Pi_{T}(\omega, k)=\frac{3}{2} m_{p h}^{2} \frac{\omega^{2}}{k^{2}}\left[1-\left(1-\frac{k^{2}}{\omega^{2}}\right) \frac{\omega}{2 k} \ln \frac{\omega+k}{\omega-k}\right](7)
\end{aligned}
$$

where $m_{p h}=e T / 3$ is called the effective photon mass. For $T=10 \mathrm{MeV}$ we get $m_{p h}=1 \mathrm{MeV}$.

The crucial quantity from which the collective phenomena are derived is the dielectric tensor relating the macroscopic electric field $D_{i}$ in the medium to the external field $E_{i}(i=x, y, z)$, i.e. in momentum space

$$
D_{i}(\omega, \mathbf{k})=\sum_{j} \epsilon_{i j}(\omega, \mathbf{k}) E_{j}(\omega, \mathbf{k})
$$

In the case of an isotropic medium it depends only on $\omega$ and on $k$ and has two independent components

$$
\epsilon_{i j}(\omega, k)=\epsilon_{T}(\omega, k)\left(\delta_{i j}-\frac{k_{i} k_{j}}{k^{2}}\right)+\epsilon_{L}(\omega, k) \frac{k_{i} k_{j}}{k^{2}} .
$$

The dielectric tensor is closely related to the polarization tensor or photon self-energy by [11]

$$
\begin{aligned}
& \epsilon_{L}(\omega, k)=1-\frac{\Pi_{L}(\omega, k)}{k^{2}}, \\
& \epsilon_{T}(\omega, k)=1-\frac{\Pi_{T}(\omega, k)}{\omega^{2}},
\end{aligned}
$$

where $\Pi_{L}$ and $\Pi_{T}$ are the longitudinal and transverse components of the polarization tensor, respectively.

The dielectric functions following from (10) and (7) can also be derived from the classical Vlasov equation together with the Maxwell equations [12], since the hightemperature limit corresponds to the classical limit.

The dispersion relations of collective plasma modes, i.e. propagation of electromagnetic waves in the plasma, can be found by using the Maxwell equation, leading to

$$
\begin{aligned}
\epsilon_{L}(\omega, k) & =0, \\
\epsilon_{T}(\omega, k) & =\frac{k^{2}}{\omega^{2}} .
\end{aligned}
$$

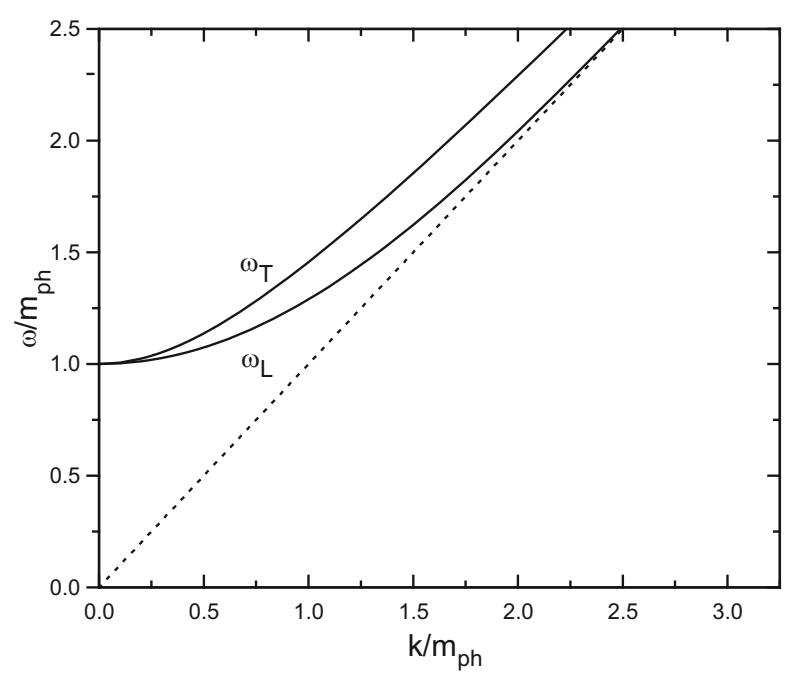

Fig. 2. Photon dispersion relation.

Combining (10), (7), and (11) gives the dispersion relations $\omega_{L, T}(k)$ of the transverse as well as longitudinal plasma waves as shown in Figure 2. The longitudinal branch, which does not exist in vacuum, is called plasmon as in the case of non-relativistic plasmas. The transverse branch does not play a role in non-relativistic plasmas but is equally important as the longitudinal one in relativistic plasmas. Both branches start at the plasma frequency $\omega_{p l}=\omega_{L, T}(k=0)=m_{p h}$. Consequently the collective plasma waves have soft momenta of the order $e T$. At high momenta $k \gg m_{p h}$ the transverse mode approaches the free dispersion $\omega_{T}=k$, corresponding to a real photon in vacuum, whereas the longitudinal mode disappears, i.e. its spectral strength is exponentially suppressed. For $T=10 \mathrm{MeV}$ we find $\omega_{p l}=1.5 \times 10^{21} \mathrm{~s}^{-1}$.

Another important quantity which can be derived from the polarization or dielectric tensor is the Debye screening length, entering the Yukawa potential of a heavy, nonrelativistic test charge in the EPP. The Debye screening length is given by the static limit of the longitudinal component of the polarization tensor $1 / \Pi_{L}(\omega=0)$, leading to $\lambda_{D}=1 /\left(\sqrt{3} m_{p h}\right)$, which is $1.1 \times 10^{-13} \mathrm{~m}$ for $T=10 \mathrm{MeV}$.

Finally from (7) we see that the polarization tensor and the dielectric function become imaginary for $\omega^{2}<k^{2}$, i.e. below the light cone $\omega=k$, corresponding to a collisionless dissipation following from the Vlasov approach, which is known as Landau damping in the non-relativstic case [5]. We also observe that the plasma waves calculated at lowest order perturbation theory are undamped since they are located at $\omega>k$.

A complete new phenomenon that does not appear in non-relativistic plasmas is the existence of fermionic plasma waves because all fermion masses are much too large in the non-relativistic case. Their dispersion relations follow from the pole of the electron propagator containing the electron self-energy. Using again the high temperature approximation for the one-loop electron self-energy of Figure 3 leads to $\left(P=\left(p_{0}, \mathbf{p}\right), p=|\mathbf{p}|\right)[9,13]$

$$
\Sigma(P)=-a\left(p_{0}, p\right) P^{\mu} \gamma_{\mu}-b\left(p_{0}, p\right) \gamma_{0}
$$




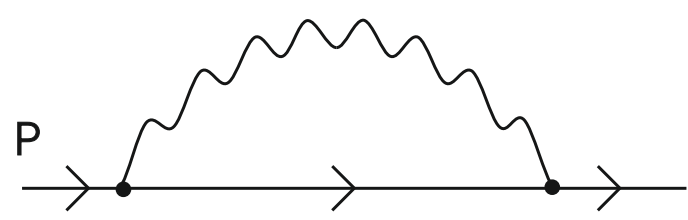

Fig. 3. One-loop electron self-energy.

with

$$
\begin{aligned}
& a\left(p_{0}, p\right)=\frac{1}{4 p^{2}}\left[\operatorname{tr}\left(P^{\mu} \gamma_{\mu} \Sigma\right)-p_{0} \operatorname{tr}\left(\gamma_{0} \Sigma\right)\right] \\
& b\left(p_{0}, p\right)=\frac{1}{4 p^{2}}\left[P^{2} \operatorname{tr}\left(\gamma_{0} \Sigma\right)-p_{0} \operatorname{tr}\left(P^{\mu} \gamma_{\mu} \Sigma\right)\right]
\end{aligned}
$$

where the traces over the $\gamma$ matrices are given by

$$
\begin{aligned}
\operatorname{tr}\left(P^{\mu} \gamma_{\mu} \Sigma\right) & =4 m_{F}^{2}, \\
\operatorname{tr}\left(\gamma_{0} \Sigma\right) & =2 m_{F}^{2} \frac{1}{p} \ln \frac{p_{0}+p}{p_{0}-p}
\end{aligned}
$$

with the effective electron mass $m_{F}=e T / \sqrt{8}$, which is $1.1 \mathrm{MeV}$ at $T=10 \mathrm{MeV}$.

The full electron propagator in the helicity representation is given by [14]

$$
S^{\star}(P)=\frac{1}{2 D_{+}(P)}\left(\gamma_{0}-\hat{p} \gamma\right)+\frac{1}{2 D_{-}(P)}\left(\gamma_{0}+\hat{p} \gamma\right),
$$

where

$D_{ \pm}(P)=-p_{0} \pm p+\frac{1}{4 p}\left[ \pm \operatorname{tr}\left(P^{\mu} \gamma_{\mu} \Sigma\right)-\left( \pm p_{0}-p\right) \operatorname{tr}\left(\gamma_{0} \Sigma\right)\right]$.

The dispersion relations following from the pole of this propagator are shown in Figure 4. Again two branches show up, one with a positive ratio of the helicity to chirality $\left(\omega_{+}\right)$following from $D_{+}=0$, the other one with a negative ratio $\left(\omega_{-}\right)$following from $D_{-}=0$, called plasmino [14]. The plasmino branch $\omega_{-}$, which does not exist in vacuum, shows an interesting behavior, namely a minimum at $k=0.41 m_{F}$, which may lead to van Hove singularities $[14,15]$. Whether these van Hove singularities will lead to observable effects in the EPP, e.g. in the electron spectrum, is a very interesting question which should be investigated in detail. It could open the exciting possibility to observe a new collective plasma wave, the plasmino, experimentally in a laser induced EPP.

\subsection{Transport properties}

Now we want to consider the interaction and properties of particles in the plasma with hard momenta, i.e. of the order of $T$ or larger. In particular we are interested in damping and transport rates, mean free paths, collision times, energy losses of these particles and other transport properties such as the shear viscosity of the EPP.

It was shown by Braaten and Pisarski [16] that a consistent treatment of gauge theories such as QED at finite

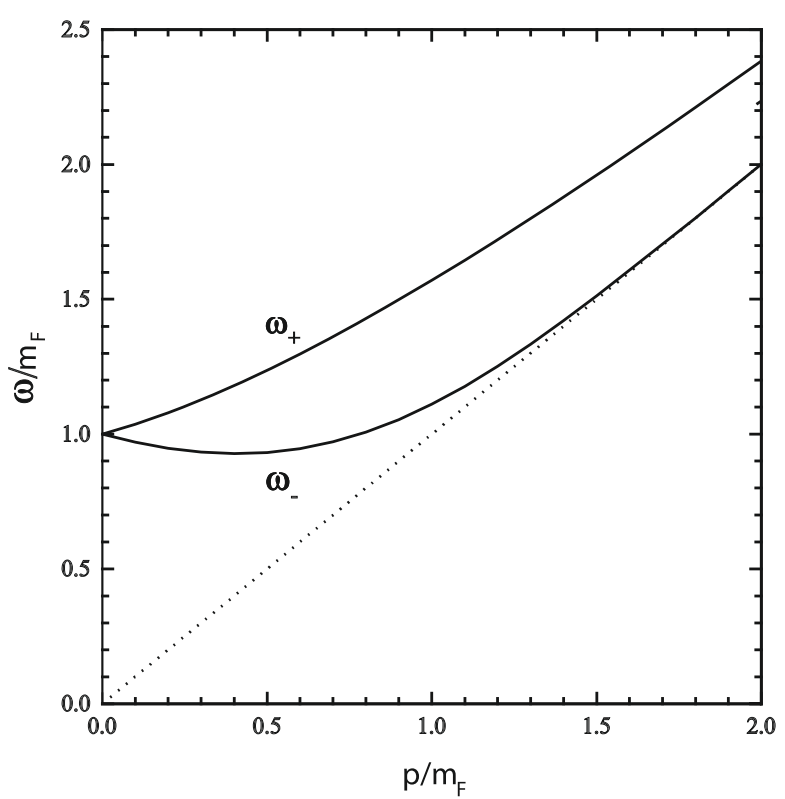

Fig. 4. Electron dispersion relation.

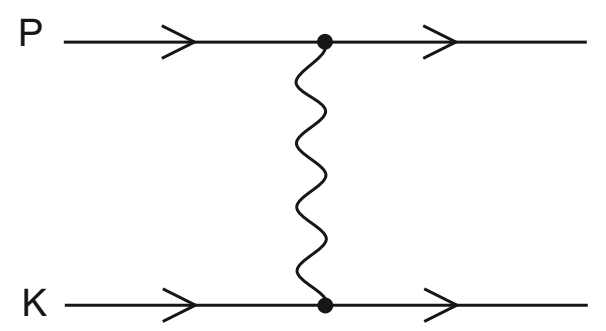

Fig. 5. Lowest order diagram for electron-electron scattering.

temperature, i.e. for obtaining results that are gauge independent, infrared finite, and complete to leading order, require the use of an effective perturbation theory using resummed Green functions based on the hard thermal loop (HTL) approximation (HTL resummation technique).

The damping rate of an electron or positron in the EPP is defined as the imaginary part of the dispersion relation $\omega_{L, T}(p)$. To lowest order it follows from the elastic scattering diagram of Figure 5. In the case of a hard electron or positron with momenta of the order of $T$ or higher it exhibits a quadratic infrared (IR) divergence which can be reduced to a logarithmic one using a HTL resummed photon propagator. This logarithmic singularity is expected to be cut-off by higher order contributions leading to [8]

$$
\gamma_{e}=\frac{e^{2} T}{4 \pi} \ln \frac{1}{e}
$$

within logarithmic accuracy, i.e. the constant under the logarithm is not determined. For $T=10 \mathrm{MeV}$ we obtain $\gamma_{e}=86 \mathrm{keV}$, which is much smaller than $\omega_{p l}=1 \mathrm{MeV}$, showing that the EPP is not overdamped.

Physically more important are the transport rates $\Gamma$ which are related to the mean free path and collision time of electrons and positrons in the EPP. They differ from the damping rate in cutting off the long range interactions with small scattering angles $\theta$ by a factor $(1-\cos \theta)$ under 

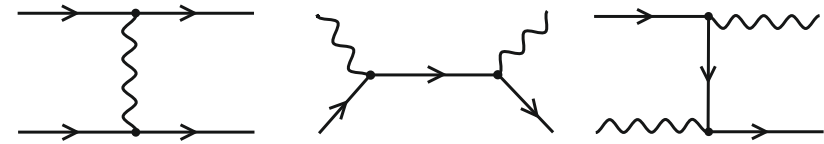

Fig. 6. Diagrams defining the collisional energy loss.

the integral defining the rate [5]. This leads to an improvement of the IR behavior (logarithmic instead of quadratic singularity in perturbation theory) and a finite result using the HTL method. Logarithmic divergent quantities can be treated consistently by splitting them into a soft part and a hard part, where the soft part is calculated using the HTL resummation technique [17]. For the transport rate we find to logarithmic accuracy

$$
\Gamma_{e}=\frac{e^{4} T^{3}}{3 \pi s} \ln \frac{1}{e}
$$

where the Mandelstam variable $s=(P+K)^{2}$ is the square of the sum of the four momenta of the incoming particles in the scattering diagram of Figure 5 . For thermal particles we replace $s$ by its thermal average $\langle s\rangle=2\langle p\rangle_{e}\langle k\rangle_{e} \simeq$ $19.3 T^{2}$, where $\langle p\rangle_{e}=\langle k\rangle_{e}=\epsilon_{e}^{e q} / \rho_{e}^{e q}=3.11 T$. Assuming again $T=10 \mathrm{MeV}$, we get $\Gamma_{e}=0.54 \mathrm{keV}$.

The mean free path $\lambda_{e}^{m f p}$ and collision time $\tau_{e}$ of the plasma particles (electrons and positrons) are given by the inverse of the transport rate $1 / \Gamma_{e}$, leading to $\lambda_{e}^{m f p}=$ $0.37 \mathrm{~nm}$ and $\tau_{e}=1.2 \times 10^{-18} \mathrm{~s}$ at $T=10 \mathrm{MeV}$.

In a non-relativistic plasma the shear viscosity can be estimated from elementary kinetic theory as [18]

$$
\eta_{i}=\frac{1}{3} \sum_{i} \rho_{i}\left\langle p_{i}\right\rangle \lambda_{i}^{m f p}
$$

where the sum is performed over the various components of the system. In an relativistic plasma the coefficient $1 / 3$ should be replaced by $4 / 15$ [19]. Using the mean free path following from (18), the density of (3), and the thermal momentum $\langle p\rangle_{e}=3.11 T$, the shear viscosity is given by (within logarithmic accuracy)

$$
\eta_{e}=\frac{55.8 T^{3}}{e^{4} \ln (1 / e)}
$$

At $T=10 \mathrm{MeV}$ the shear viscosity coefficient is $\eta_{e}=$ $7.9 \times 10^{10} \mathrm{~Pa} \mathrm{~s}$.

Another quantity of interest in a plasma is its stopping power or the energy loss of an energetic particle in the plasma. There are two contributions, namely the energy loss by collisions and the radiative one by bremsstrahlung. In a relativistic plasma the latter one becomes important. The collisional energy loss is given by the mean energy transfer divided by the mean free path leading to [20]

$$
\frac{d E}{d x}=\frac{1}{v} \int d \gamma \omega
$$

where $v$ is the particle velocity, $\gamma$ the damping or interaction rate proportional to the plasma density and the
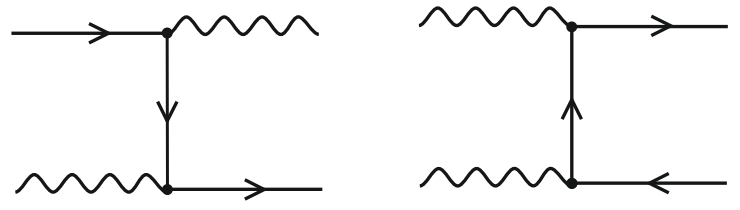

Fig. 7. Diagrams defining the photon damping rate.

collision cross section, and $\omega$ the energy transfer from the energetic particle to the plasma particle in the collision. Using for the collision cross section the lowest order diagrams in Figure 6, the collisional energy of a muon with mass $M$ in an EPP has been calculated by Braaten and Thoma [20] applying the HTL resummation technique

$\frac{d E}{d x}=\frac{e^{4} T^{2}}{24 \pi}\left(\frac{1}{v}-\frac{1-v^{2}}{2 v^{2}} \ln \frac{1+v}{1-v}\right)\left(\ln \frac{E}{M}+\ln \frac{1}{e}+A(v)\right)$,

where $A(v)$ is a slowly varying function of the muon velocity $v$ between 1.3 and 1.5 .

The collisional energy loss of an electron with energy $E \gg T$ is approximately given by [20]

$$
\frac{d E}{d x}=\frac{e^{4} T^{2}}{48 \pi} \ln \frac{15.3 E}{e^{2} T}
$$

This leads to an energy loss of $200 \mathrm{MeV} / \mathrm{nm}$ for an electron or positron with an energy of $E=100 \mathrm{MeV}$ at $T=10 \mathrm{MeV}$, showing that such an electron is stopped (thermalized) within a fraction of a nanometer. So far no calculations of the radiative energy loss in an EPP have been performed to our knowledge.

The damping rate of a photon in an EPP follows from the diagram in Figure 7, where a HTL resummed electron propagator has to be used in case of soft momenta of the exchanged electron (positron). In contrast to the electron damping rate, the photon rate is infrared finite using the HTL method due to the presence of an electron propagator in Figure 7 instead of the photon propagator in Figure 5. Hence there is no need to cut off the long range interaction introducing a transport cross section. The result for a photon with energy $E=p$ reads [21]

$$
\Gamma_{p h}=\frac{e^{4} T^{2}}{64 \pi E} \ln \frac{3.88 E}{e^{2} T} .
$$

The mean free path and the collision time of photons in an EPP are given by $1 / \Gamma_{p h}$. For a thermal photon with the mean momentum $\langle p\rangle_{p h}=\epsilon_{p h}^{e q} / \rho_{p h}^{e q}=2.75 T$ at $T=$ $10 \mathrm{MeV}$ the mean free path $\lambda_{p h}^{m f p}=0.28 \mathrm{~nm}$ and the collision time $\tau_{p h}=9.4 \times 10^{-19} \mathrm{~s}$ follow. Actually the damping rate given in (24) is a lower limit as higher order effects will enlarge it. As a matter of fact, the photon production rate in a QGP, which is the inverse process of the damping rate [21], was shown to be about a factor of 2 larger taking bremsstrahlung into account [22].

For the viscosity of the photon component using the above mean free path, the photon density (see above), and 
Table 1.

\begin{tabular}{lll}
\hline Quantity & Formula & Value at T=10 MeV \\
\hline Electron-positron density & $\rho_{e}^{e q}=3 / \pi^{2} \zeta(3) T^{3}$ & $4.9 \times 10^{40} \mathrm{~m}^{-3}$ \\
Photon density & $\rho_{p h}^{e q}=2 / \pi^{2} \zeta(3) T^{3}$ & $3.2 \times 10^{40} \mathrm{~m}^{-3}$ \\
Electron-positron energy density & $\epsilon_{e}^{e q}=7 \pi^{2} / 60 T^{4}$ & $2.4 \times 10^{29} \mathrm{~J} \mathrm{~m}^{-3}$ \\
Photon energy density & $\epsilon_{p h}^{e q}=\pi^{2} / 15 T^{4}$ & $1.4 \times 10^{29} \mathrm{~J} \mathrm{~m}^{-3}$ \\
Total energy density & $\epsilon^{e q}=11 \pi^{2} / 60 T^{4}$ & $3.8 \times 10^{29} \mathrm{~J} \mathrm{~m}^{-3}$ \\
Thermal electron momentum & $\langle p\rangle_{e}=\epsilon_{e}^{e q} / \rho_{e}^{e q}=3.11 T$ & $31 \mathrm{MeV}$ \\
Thermal photon momentum & $\langle p\rangle_{p h}=\epsilon_{p h}^{e q} / \rho_{p h}^{e q}=2.75 T$ & $28 \mathrm{MeV}$ \\
Interparticle distance & $d \simeq \rho_{e}^{e q}-1 / 3$ & $2.7 \times 10^{-14} \mathrm{~m}$ \\
Coulomb coupling parameter & $\Lambda=e^{2} /(d T)$ & $5.3 \times 10^{-3}$ \\
Effective photon mass & $m_{p h}=e T / 3$ & $1 \mathrm{MeV}$ \\
Plasma frequency & $\omega_{p l}=m_{p h}$ & $1.5 \times 10^{21} \mathrm{~s}^{-1}$ \\
Debye screening length & $\lambda_{D}=1 /\left(\sqrt{3} m_{p h}\right)$ & $1.1 \times 10^{-13} \mathrm{~m}$ \\
Effective electron mass & $m_{F}=e T /(2 \sqrt{2})$ & $1.1 \mathrm{MeV}$ \\
Electron damping rate & $\gamma_{e}=e^{2} T /(4 \pi) \ln (1 / e)$ & $86 \mathrm{keV}$ \\
Electron transport rate & $\Gamma_{e}=e^{4} T^{3} /(3 \pi s) \ln (1 / e)$ & $0.54 \mathrm{keV} \mathrm{for} s=19.3 T^{2}$ \\
Photon damping rate & $\Gamma_{p h}=e^{4} T^{2} /(64 \pi E) \ln \left(3.88 E / e^{2} T\right)$ & $0.70 \mathrm{keV} \mathrm{for} \mathrm{E=2.75T}$ \\
Electron mean free path & $\lambda_{e}^{m f p}=1 / \Gamma_{e}$ & $0.37 \mathrm{~nm}$ \\
Photon mean free path & $\lambda_{p h}^{m f p}=1 / \Gamma_{p h}$ & $0.28 \mathrm{~nm}$ \\
Electron collision time & $\tau_{e}=1 / \Gamma_{e}$ & $1.2 \times 10^{-18} \mathrm{~s}$ \\
Photon collision time & $\tau_{p h}=1 / \Gamma_{p h}$ & $9.4 \times 10^{-19} \mathrm{~s}$ \\
Electron viscosity & $\eta_{e}=55.8 T^{3} /\left[e^{4} \ln (1 / e)\right]$ & $7.9 \times 10^{10} \mathrm{~Pa} \mathrm{~s}$ \\
Photon viscosity & $\eta_{p h}=48.7 T^{3} /\left[e^{4} \ln (3.27 / e)\right]$ & $3.5 \times 10^{10} \mathrm{~Pa} \mathrm{~s}$ \\
Total viscosity & $\eta=\eta_{e}+\eta_{p h}$ & $(1.1-1.6) \times 10^{11} \mathrm{~Pa} \mathrm{~s}$ \\
Electron energy loss & $d E / d x=e^{4} T^{2} /(48 \pi) \ln \left(15.3 E / e^{2} T\right)$ & $200 \mathrm{MeV} / \mathrm{nm}$ for $E=100 \mathrm{MeV}$ \\
\hline
\end{tabular}

the mean photon energy $\langle p\rangle_{p h}=2.75 T$ we find

$$
\eta_{p h}=\frac{48.7 T^{3}}{e^{4} \ln (3.27 / e)}
$$

corresponding to $3.5 \times 10^{10} \mathrm{~Pa}$ s at $T=10 \mathrm{MeV}$. Hence the viscosity of the $\operatorname{EPP} \eta=\eta_{e}+\eta_{p h}$ has similar contributions from the electrons and photons.

A more advanced calculation of the total viscosity of the EPP based on the Kubo formula yields within logarithmic accuracy [23]

$$
\eta=\frac{188 T^{3}}{e^{4} \ln (1 / e)} .
$$

This result is about a factor of 1.5 larger than the one presented here based on the elementary kinetic theory, which is typically valid within a factor of 2 [18].

A summary of the QED results of the EPP properties discussed above is presented in Table 1.

\subsection{Non-equilibrium and finite chemical potential}

EPPs produced in strong laser fields are probably not in complete equilibrium. For example, it has been predicted by Shen and Meyer-ter-Vehn [2] that a positron density of about $5 \times 10^{28} \mathrm{~m}^{-3}$ at a temperature of $10 \mathrm{MeV}$ can be reached. This density deviates from the equilibrium density (3) by 12 orders of magnitude. In the following we will therefore assume that the EPP produced by lasers is in thermal but not in chemical equilibrium. Then we can replace the distribution functions for the electrons and positrons by Fermi-Dirac distributions multiplied by a fugacity factor $\lambda$ describing the deviation from chemical equilibrium, $f_{F}(p)=\lambda n_{F}(p)$. This assumption has been used for example for describing the chemical equilibration of the QGP in ultrarelativistic heavy-ion collisions [24]. The fugacity is given by the ratio of the experimental to equilibrium particle density, since the experimental density follows from integrating over the non-equilibrium distribution, i.e.

$$
\rho_{e x p}=g_{F} \int \frac{d^{3} p}{(2 \pi)^{3}} \lambda n_{F}(p)=\lambda \rho_{e q} \Rightarrow \lambda=10^{-12} .
$$

Using the real time formalism, QED perturbation theory and the HTL method can also be extended to nonequilibrium situations like the one discussed above [25]. For example, the effective photon mass is given now by

$$
m_{p h}^{2}=\frac{4 e^{2}}{3 \pi^{2}} \int_{0}^{\infty} d p p f_{F}(p) .
$$

For $T=10 \mathrm{MeV}$ we then find for the non-equilibrium photon mass $m_{p h}^{n o n e q}=\sqrt{\lambda} m_{p h}=1 \mathrm{eV}$ and the plasma frequency $\omega_{p l}^{n o n e q}=1.5 \times 10^{15} \mathrm{~Hz}$. The Debye screening length in such an EPP is $\lambda_{D}=0.1 \mu \mathrm{m}$. In order to speak of a plasma with collective behavior its dimension $L$ should be much larger than $\lambda_{D}$, i.e. at least of the order of $1 \mu \mathrm{m}$.

Note also that the mean free path of photons, which is inversely proportional to the electron-positron density, is much larger than the extension of the plasma droplet in such a dilute EPP. Therefore photons escape from the 


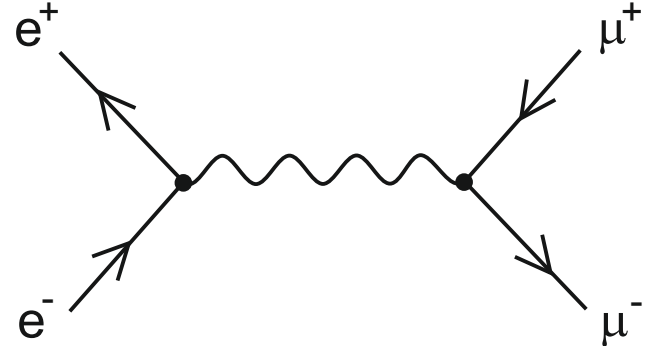

Fig. 8. Lowest order contribution of the muon production.

plasma without interaction and may serve as a probe for the interior and evolution of the EPP similar to photons from a quark-gluon plasma fireball in an ultrarelativistic plasma [27].

Furthermore, an anisotropic EPP can also be described by quantum field theoretic methods [26]. In this case instabilities can occur [28].

Finally a possible difference between the positron density and the electron density can be treated by introducing a finite chemical potential $\mu$, i.e. using the distribution

$$
n_{F}(p)=\frac{1}{e^{(p \pm \mu) / T}+1}
$$

for the electrons (negative sign) and positrons (positive sign). Such a difference comes from the fact that the laser produced EPP is embedded in a hot electron and cold ion background of the target. Therefore there will be an excess of electrons over positrons in the hot, relativistic EPP. The methods described above, such as the HTL resummation, can be generalized easily to this case [29]. For example, the energy density is given by

$$
\epsilon^{e q}=\frac{11 \pi^{2}}{60} T^{4}+\frac{1}{2} T^{2} \mu^{2}+\frac{1}{4 \pi^{2}} \mu^{4}
$$

or the effective photon energy by

$$
m_{p h}^{2}=\frac{e^{2} T^{2}}{9}\left(1+\frac{3 \mu^{2}}{\pi^{2} T^{2}}\right) .
$$

\subsection{Particle production}

At high temperatures above $10 \mathrm{MeV}$ also other particle species will be produced, e.g. muons with a mass of $m_{\mu}=106 \mathrm{MeV}$. Their rate follows to lowest order from the diagram in Figure 8 (Born term). We assume that $m_{e} \ll T \ll m_{\mu}$ holds. The first inequality implies that the electron mass can be put to zero and the latter inequality implies that muons are not equilibrated. Then the muon production rate to lowest order $\left(e^{-} e^{+} \rightarrow \gamma^{*} \rightarrow \mu^{-} \mu^{+}\right)$is given by (for more details see Ref. [3])

$$
\begin{aligned}
\frac{d N}{d^{4} x d^{4} p} & =\frac{\alpha^{2}}{24 \pi^{4}}\left(1+\frac{2 m_{\mu}^{2}}{M^{2}}\right)\left(1-\frac{4 m_{\mu}^{2}}{M^{2}}\right)^{1 / 2} \frac{T}{p} \\
& \times \frac{1}{\exp (E / T)-1} \ln \frac{1+\exp [-(E+p) /(2 T)]}{1+\exp [-(E-p) /(2 T)]}
\end{aligned}
$$

where $M^{2}=E^{2}-p^{2}$ is the invariant mass of the virtual photon $\gamma^{*}, E$ its energy and $p=|\mathbf{p}|$ its momentum. Because of $M^{2}=E^{2}-p^{2}>4 m_{\mu}^{2}$ the rate is suppressed exponentially for temperatures below $2 m_{\mu}$.

In order to obtain the spectrum from this formula one has to integrate over the space-time volume, taking into account the space-time evolution by using, for example, a hydrodynamical model. The total muon yield then follows from integrating the spectrum over the energy and momentum of the virtual photon.

At temperatures above $10 \mathrm{MeV}$ also hadron production becomes important, in particular pion production [30].

\section{Conclusions}

The aim of this presentation is the prediction of properties of ultrarelativistic thermalized EPPs produced in laser fields or supernovae. The ultrarelativistic EPP is a weakly coupled system. Therefore its equation of state can be described in first approximation by an ideal ultrarelativistic gas. Interactions within the EPP can be described by perturbative QED at finite temperature. In this way collective phenomena (plasma waves, Debye screening) and transport properties (damping rates, mean fee paths, relaxation times, production rates, viscosity, energy loss) can be computed. A complete new phenomenon, fermionic plasma waves (plasmino), which is absent in non-relativistic plasmas, might be observable by van Hove singularities. The deviation from chemical equilibrium, as expected for laser produced EPPs, can also be treated by extending QED to the non-equilibrium case.

I would like to thank D. Habs, J. Rafelski, I. Kouznetsova, and G. Moore for helpful discussions and hints. The author acknowledges the support by the European Commission under contract ELI pp 212105 in the framework of the program FP7 Infrastructures-2007-1.

\section{References}

1. S.J. Hardy, M.H. Thoma, Phys. Rev D 63, 025014 (2001)

2. B. Shen, J. Meyer-ter-Vehn, Phys. Rev. E 65, 016405 (2001)

3. M.H. Thoma, e-print ArXiv:0801.0956

4. S. Ichimaru, Rev. Mod. Phys. 54, 1017 (1982)

5. E.M. Lifshitz, L.P. Pitaevskii, Physical Kinetics (Pergamon Press, Oxford, 1981)

6. J.I. Kapusta, Finite Temperature Field Theory (Cambridge University Press, New York, 1989)

7. N.P. Landsmann, C.G. van Weert, Phys. Rep. 145, 141 (1987)

8. M.H. Thoma, in Quark-Gluon Plasma 2, edited by R.C. Hwa (World Scientific, Singapore, 1995), p. 51

9. V.V. Klimov, Zh. Eksp. Teor. Fiz. 82, 336 (1982) [Sov. Phys. JETP 55, 199 (1982)]

10. H.A. Weldon, Phys. Rev. D 26, 1394 (1982) 
11. H.T. Elze, U. Heinz, Phys. Rep. 183, 81 (1989)

12. V.P. Silin, Z. Eksp. Teor. Fiz. 38, 1577 (1960) [Sov. Phys. JETP 11, 1136 (1960)]

13. H.A. Weldon, Phys. Rev. D 26, 2789 (1982)

14. E. Braaten, R.D. Pisarski, T.C. Yuan, Phys. Rev. Lett. 64, $2242(1990)$

15. A. Peshier, M.H. Thoma, Phys. Rev. Lett. 84, 841 (2000)

16. E. Braaten, R.D. Pisarski, Nucl. Phys. B 337, 569 (1990)

17. E. Braaten, T.C. Yuan, Phys. Rev. Lett. 66, 2183 (1991)

18. F. Reif, Fundamentals of Statistical and Thermal Physics (McGraw-Hill, New York, 1965)

19. S.R. de Groot, W.A. van Leeuwen, C.G. van Weert, Relativistic Kinetic Theory (North-Holland, Amsterdam, 1980)

20. E. Braaten, M.H. Thoma, Phys. Rev. D 44, 1298 (1991)

21. M.H. Thoma, Phys. Rev D 51, 862 (1995)
22. P. Arnold, G.D. Moore, L.G. Yaffe, J. High Energy Phys. 112, 9 (2001)

23. P. Arnold, L.G. Yaffe, G.D. Moore, J. High Energy Phys. 11, $1(2000)$

24. T.S. Biró, E. van Doorn, B. Müller, M.H. Thoma, X.N. Wang, Phys. Rev. C 48, 1275 (1993)

25. M.E. Carrington, H. Defu, M.H. Thoma, Eur. Phys. J. C 67, 347 (1999)

26. S. Mrówczyński, M.H. Thoma, Phys. Rev. D 62, 036011 (2000)

27. T. Peitzmann, M.H. Thoma, Phys. Rep. 364, 175 (2002)

28. J. Randrup, S. Mrówczyński, Phys. Rev. C 68, 034909 (2003)

29. H. Vija, M.H. Thoma, Phys. Lett. B 342, 212 (1995)

30. I. Kuznetsova, D. Habs, J Rafelski, Phys. Rev. D 78, 014027 (2008) 